\title{
COMPARAÇÃo DE MÉTODOS DE EXTRAÇÃO DE COBRE, ZINCO, FERRO E MANGANÊS EM SOLOS DO ESTADO DO RIO DE J ANEIRO(1)
}

\author{
M. G. PEREIRA(2), D. V. PÉ REZ ${ }^{(3)}$, G. S. VALLADARES $^{(4)}$, \\ J. M. P. F. SOUZA(5) \& L. H. C. ANJ OS ${ }^{(2)}$
}

\begin{abstract}
RESUMO
As conseqüências da deficiência ou toxidez de micronutrientes na produção agrícola do estado do Rio de J aneiro são pouco conhecidas. Desta forma, em 1997, foram quantificados os teores de cobre, zinco, ferro e manganês em 103 amostras de horizontes superficiais de solos representativos do estado, sendo utilizados os extratores Mehlich-1, DTPA-TEA e HCl 0,1 mol L-1. De maneira geral, o extrator $\mathrm{HCl}$ 0,1 mol L-1 foi o que apresentou maior poder de extração dos micronutrientes estudados, tendo o Mehlich-1 extraído as menores quantidades de cobre e ferro e o DTPA-TEA as menores quantidades de zinco e manganês. Segundo os resultados, equações de conversão entre extratores, para um mesmo elemento, e a utilização de faixas de interpretação desenvolvidas para regiões edáficas diferentes das condições do trabal ho podem não estimar corretamente a disponibilidade dos micronutrientes às plantas.
\end{abstract}

Termos de indexação: micronutrientes, fertilidade dos solos, extratores.

(1) Apresentado no XVI Congresso Brasileiro de Ciência do Solo, Rio de J aneiro (RJ ), 20 a 26 de julho de 1997. Recebido para publicação em abril de 1998 e aprovado em março de 2001.

(2) Professor do Departamento de Solos da Universidade Federal Rural do Rio de J aneiro - UFRRJ . CEP 23890-000 Seropédica (RJ ). E-mail: gervasio@ufrrj.br

(3) Pesquisador da EMBRAPA/CNPS, Rua J ardim Botânico 1024. CEP 22460-000 Rio de J aneiro (RJ ).

(4) Doutorando do Curso de Pós-Graduação em Agronomia - Ciência do Solo do Departamento de Sol os, UFRRJ. Bolsista da CAPES.

(5) Mestrando do Curso de Pós-Graduação em Agronomia - Ciência do Solo. Departamento de Solos, UFRRJ. Bolsista da CAPES e CNPq/PIBIC. 


\title{
SUMMARY: COMPARISON OF EXTRACTION METHODS FOR COPPER, ZINC, IRON AND MANGANESE IN SOILS OF THE STATE OF RIO DE J ANEIRO, BRAZIL
}

\begin{abstract}
Theconsequences of micronutrient deficiency or toxicity for theagricultural productivity in the state of Rio deJ ane ro are not adequately known. For this reason, in 1997, thelevels of copper, zinc, iron, and manganese were quantified in 103 samples from surface horizons of representative soils of the state The extractant solutions used were Mehlich-1, DTPATEA, and $\mathrm{HCl} 0.1 \mathrm{~mol} \mathrm{~L}^{-1}$. In general, the $\mathrm{HCl} 0.1 \mathrm{~mol} \mathrm{~L}^{-1}$ solution extracted the highest amounts of the studied mi cronutrients. Mehlich-1 extracted the smal lest amounts of copper and iron, and the DTPA-TEA extracted the smallest amounts of zinc and manganese The results showed that the use of linear regression equations for conversion of values amongst methods of a specific el ement and the use of ranges of interpretation developed in different edaphic conditions, in comparison to the present work, are not recommended. They can lead to incorrect diagnoses.
\end{abstract}

Index terms: micronutrients, soil fertility, extractant solution.

\section{INTRODUÇÃO}

Alguns elementos essenciais às plantas são absorvidos em pequenas quantidades, sendo, por isso, denominados micronutrientes. Diversos fatores afetam a quantidade e a disponibilidade desses nutrientes nos solos. A despeito do menor consumo, sucessivos anos de utilização agrícola levam à exaustão das reservas desses el ementos, que devem, então, ser repostos (Camargo, 1988).

A avaliação dos micronutrientes no solo é fundamental e o sucesso desta está associado à escol ha adequada do processo de extração (Ribeiro \& Sarabia, 1984; Abreu, 1995). Uma das maneiras de avaliar a possibilidade de utilização de diversos tipos de extratores na análise de rotina, segundo Eckert \& Watson (1997), está na correlação, em ensaios de laboratório, das quantidades extraídas pelo novo extrator proposto e o tradicionalmente usado. Nesse sentido, os trabal hos deWolf \& Baker (1985), J ones \& Piha (1989), Sims (1989), Alva (1993), Mamo et al. (1996) e Garcia et al. (1997) são exemplos da tentativa de alguns países em estabelecer um extrator, para as suas rotinas de análise de solo, por meio da comparação de métodos de extração.

NoBrasil, segundo Cantarella et al . (1994), mais de $50 \%$ dos laboratórios em atividade, no período de 1982-89, utilizavam o Mehlich-1 $\left(\mathrm{HCl} 0,05 \mathrm{~mol} \mathrm{~L}^{-1} \mathrm{e}\right.$ $\mathrm{H}_{2} \mathrm{SO}_{4}$ 0,0125 mol L-1) como extrator de micronutrientes. Atual mente, para avaliar a disponibilidade dos micronutrientes em solos, os diversos laboratórios do Brasil empregam as soluções de Mehlich-1, $\mathrm{H}_{2} \mathrm{SO}_{4}$, DTPA, água, $\mathrm{CaCl}_{2}, \mathrm{KCl}$, $\mathrm{Mg}\left(\mathrm{NO}_{3}\right)_{2}, \mathrm{Ca}\left(\mathrm{NO}_{3}\right)_{2}$ e $\left(\mathrm{NH}_{4}\right)_{2} \mathrm{C}_{2} \mathrm{O}_{4}$ (Abreu, 1995).

NoBrasil, as mel hores correlações entre os teores de $\mathrm{Cu}, \mathrm{Fe}, \mathrm{Mn}$ e $\mathrm{Zn}$ em solos e os teores desses nas plantas têm sido obti das para o método que emprega a solução DTPA-TEA como extratora (Abreu, 1995; Cantarella et al., 1998). As soluções Mehlich-1 eácido dorídrico, utilizadas emal guns estados, apresentaram resultados iguais ou inferiores aos obtidos com a solução de DTPA-TEA (Ribeiro \& Sarabia, 1984; Abreu, 1995).

No estado do Rio de J aneiro, existem poucas informações sobre a influência da deficiência ou toxidez de micronutrientes na produção agrícola. Além disso, não são conhecidos níveis críticos, nesse estado, para esses el ementos, sendo usados dados de outras regiões, para recomendações de sua aplicação.

Os objetivos deste trabalho foram: comparar a capacidade de extração dos três métodos de determinação de formas disponíveis de $\mathrm{Cu}, \mathrm{Zn}$, Fee $\mathrm{Mn}$ mais usados no Brasil erelacionar os resultados obtidos com as faixas de interpretação em uso no Brasil.

\section{MATERIAL E MÉTODOS}

No primeiro semestre de 1997, foram analisadas 103 amostras de horizontes superficiais de solos representativos do estado do Rio de J aneiro, pertencentes à col eção de solos da Embrapa Sol os e do Departamento de Solos da UFRRJ . As amostras correspondem às principais ordens de solos (EMBRAPA, 1999) do estado, assim distribuídas: 32 deArgissolos, 27 de Latossolos, 13 de Gleissolos, 10 Neossolos, 7 de Planossolos, 4 de Cambissolos, 4 de Chernossolos, 2 de Espodossolos, 2 de Organossolos, 1 de Vertissol o e 1 de Nitossolo.

$\mathrm{O}$ teor de argila variou de 10 a $760 \mathrm{~g} \mathrm{~kg}^{-1}$. As amostras foram classificadas, de acordo com Freire 
et al. (1988), como sendo $17,5 \%$ de textura arenosa (teor de argila menor que $150 \mathrm{~g} \mathrm{~kg}^{-1}$ ), 48,5\% de textura média (teor entre 150 e $350 \mathrm{~g} \mathrm{~kg}^{-1}$ ), 28,2\% de textura argilosa (teor entre 350 e $600 \mathrm{~g} \mathrm{~kg}^{-1}$ ) e $5,8 \%$ de textura muito argilosa (teor superior a $600 \mathrm{~g} \mathrm{~kg}^{-1}$ ).

Os valores de pH em água variaram de 3,5 a 7,4, assim distribuídos, segundo classificaçãoapresentada por Freire et al. (1988): 20,4\% das amostras apresentaram-se na faixa extremamente ácida (menor que 4,3), 49,5\% na fortemente ácida (de 4,4 a 5,3), 27,2\% na moderadamenteácida (de 5,4 a 6,5), $1,9 \%$ na praticamente neutra (de 6,6 a 7,3 ) e $1,0 \%$ na moderadamente alcalina $(7,4$ a 8,3).

Para determinar os micronutrientes, foram utilizados os seguintes extratores:

Solução de $\mathrm{HCl}$ 0,05 mol L ${ }^{1}+\mathrm{H}_{2} \mathrm{SO}_{4}$ 0,0125 mol L-1 (Mehlich-1): $10 \mathrm{~g}$ de solo e $50 \mathrm{~mL}$ de solução extratora, cinco minutos de agitação e dezesseis horas derepouso. Em seguida, o material foi filtrado e, no extrato, determinados os micronutrientes (Nelson et al., 1953).

Solução de $\mathrm{HCl}$ 0,1 mol L-1: $10 \mathrm{~g}$ de solo e $50 \mathrm{~mL}$ de solução extratora, cinco minutos de agitação, seguida de filtragem (Nelson et al., 1959).

Solução deDTPA-TEA: usaram-se $5 \mathrm{~g}$ de solo com $25 \mathrm{~mL}$ da solução extratora DTPA-TEA pH 7,3, agitação de duas horas e posterior filtragem (Lindsay \& N orvell, 1978).

Em todos os extratos, foi efetuada a filtração com papel de filtro Whatman 42 e a quantificação dos micronutrientes foi efetuada por espectrofotometria de absor ção atômica.

Para determinar os teores de argila e de carbono orgânico e do pH em água, usaram-se os métodos descritos em EMBRAPA (1997).

O método estatístico empregado para comparar os teores de micronutrientes obtidos a partir dos diferentes extratores foi o da regressão linear $(Y=$ $\mathrm{b}_{0}+\mathrm{b}_{1} \mathrm{X}$ ), conforme sugeriram Miller \& Miller (1993). As hipóteses nulas formuladas foram de que a declividade $\left(b_{1}\right)$ não diferiria de 1 e que o intercepto $\left(b_{0}\right)$ não diferiria de zero. Tais hipóteses foram testadas por meio do cál culo dos limites de confiança, a 95\%, para os dois coeficientes. O programa estatístico adotado na análise de dados foi oStatistical Package for Social Sciences-SPSS (Nie et al., 1979).

\section{RESULTADOS E DISCUSSÃO}

Os teores mínimos e máximos de micronutrientes extraídos pelos três métodos são apresentados no quadro 1. Comparando as faixas de val ores obtidas, verificou-se que estão em concordância com os dados encontrados e citados por Ferreira \& Cruz (1991) e Assumpção (1995) para vários sol os brasileiros.

A solução de $\mathrm{HCl}$ 0,1 mol L-1 foi a que extraiu as maiores quantidades dos quatro micronutrientes anal isados (Quadro 2), o que é indicado pel as declividades médias acima de 1,0 e, no caso do cobre, pelo interceptoacima de 0,0. Em geral, o poder de extração para os extratores e micronutrientes obedeceu à seguinte ordem: (a) para $\mathrm{Cu} \Rightarrow \mathrm{HCl}>$ DTPATEA > Mehlich-1; (b) para $\mathrm{Zn} \Rightarrow \mathrm{HCl}>$ Mehlich-1 > DTPA-TEA; (c) $\mathrm{Fe} \Rightarrow \mathrm{HCl}>$ DTPA-TEA > Mehlich-1; e (d) para $\mathrm{Mn} \Rightarrow \mathrm{HCl}>$ Mehlich-1 >DTPA-TEA.

Os coeficientes de determinação $\left(R^{2}\right)$ mais elevados foram obtidos, para os quatro el ementos anal isados, entre o $\mathrm{HCl} 0,1 \mathrm{~mol} \mathrm{~L}^{-1}$ e o Mehlich-1. Tal comportamento deveu-se às características ácidas destes extratores que promoveram a solubilização tanto das formas lábeis quanto das formas não-lábeis dos micronutrientes (Bataglia \& Raij, 1989).

A partir de al gumas observações de Sims (1989) eAbreu et al. (1997), foram testadas, também, novas regressões pela exclusão dos dados referentes às amostras que apresentavam teores de $\mathrm{Fe}, \mathrm{Mn}, \mathrm{Zn}$ e Cu acima do limite de interesse agronômico. Como o DTPA-TEA tem-se demonstrado melhor nas correlações entre os teores de $\mathrm{Fe}, \mathrm{Cu}, \mathrm{Mn}$ e $\mathrm{Zn}$ em sol os e nas plantas (A breu, 1995; Cantarella et al., 1998), resolveu-se adotar os limites das faixas de interpretação consideradas altas por Raij et al. (1996), os quais se basearam nos teores disponíveis dos mi cronutrientes estudados pel o referi do extrator. Nesse sentido, todos os valores acima de 5, 5, 1,6 e

Quadro 1. Teores mínimos e máximos $\left(\mathrm{mg} \mathrm{kg}^{-1}\right)$ dos micronutrientes extraídos pelas diferentes soluções extratoras

\begin{tabular}{lllrr}
\hline Extrator & Cobre & Zinco & Ferro & Manganês \\
\hline Mehlich-1(1) & $0,03-25,80$ & $0,14-12,69$ & $4,47-436,22$ & $0,24-185,13$ \\
DTPA-TEA(2) & $0,00-9,74$ & $0,47-19,20$ & $2,60-273,46$ & $0,00-283,75$ \\
HCl 0,1 mol L-1(3) & $0,00-39,22$ & $0,00-24,88$ & $63,64-884,62$ & $0,00-467,50$ \\
\hline
\end{tabular}

${ }^{(1)}$ Nelson et al. (1953). ${ }^{(2)}$ Lindsay \& Norvell (1978). ${ }^{(3)}$ Nelson et al. (1959). 
Quadro 2. Declividades e interceptos da regressão linear $\left(Y=b_{0}+b_{1} X\right)$ estimados entre os três tipos de extratores

\begin{tabular}{|c|c|c|c|c|c|c|c|c|}
\hline \multirow{2}{*}{$\mathbf{Y}$} & \multirow{2}{*}{$x$} & \multicolumn{3}{|c|}{ Declividade $\left(b_{1}\right)$} & \multicolumn{3}{|c|}{ Intercepto (bo) } & \multirow{2}{*}{$\mathbf{R}^{2}$} \\
\hline & & Mínimo & Médio & Máximo & Mínimo & Médio & Máximo & \\
\hline $\mathrm{Cu}^{(2)}$ & $\mathrm{Cu}^{(1)}$ & 2,02 & 2,48 & 2,94 & $-0,58$ & $-0,03$ & 0,53 & 0,53 \\
\hline $\mathrm{Cu}(3)$ & $\mathrm{Cu}(1)$ & 3,80 & 3,91 & 4,01 & $-0,41$ & $-0,29$ & $-0,16$ & 0,98 \\
\hline $\mathrm{Cu}^{(3)}$ & $\mathrm{Cu}^{(2)}$ & 0,68 & 0,84 & 1,00 & 0,15 & 0,76 & 1,37 & 0,53 \\
\hline $\mathrm{Zn}^{(2)}$ & $\mathrm{Zn}^{(1)}$ & 0,43 & 0,49 & 0,56 & 0,75 & 1,01 & 1,27 & 0,69 \\
\hline $\mathrm{Zn}^{(3)}$ & $Z n^{(1)}$ & 1,22 & 1,37 & 1,52 & $-0,82$ & $-0,21$ & 0,40 & 0,76 \\
\hline $\mathrm{Zn}^{(3)}$ & $\mathrm{Zn}^{(2)}$ & 1,78 & 2,10 & 2,42 & $-2,31$ & $-1,37$ & $-0,42$ & 0,62 \\
\hline $\mathrm{Fe}^{(2)}$ & $\mathrm{Fe}^{(1)}$ & 0,80 & 1,13 & 1,46 & 29,83 & 49,85 & 69,87 & 0,31 \\
\hline $\mathrm{Fe}^{(3)}$ & $\mathrm{Fe}^{(1)}$ & 3,04 & 3,42 & 3,79 & 51,42 & 74,07 & 96,73 & 0,76 \\
\hline $\mathrm{Fe}^{(3)}$ & $\mathrm{Fe}^{(2)}$ & 0,81 & 1,12 & 1,43 & 72,14 & 112,69 & 153,25 & 0,33 \\
\hline$M n^{(2)}$ & $M n^{(1)}$ & 0,63 & 0,70 & 0,77 & 3,77 & 8,53 & 13,29 & 0,81 \\
\hline $\mathrm{Mn}^{(3)}$ & $M n^{(1)}$ & 1,53 & 1,63 & 1,72 & 1,86 & 8,52 & 15,18 & 0,92 \\
\hline$M n^{(3)}$ & $M n^{(2)}$ & 1,83 & 2,00 & 2,17 & $-9,22$ & 0,82 & 10,87 & 0,84 \\
\hline
\end{tabular}

(1) Mehlich-1. ${ }^{(2)}$ DTPA-TEA. ${ }^{(3)} \mathrm{HCl} \mathrm{0,1} \mathrm{mol} \mathrm{L-1.}$

$0,8 \mathrm{mg} \mathrm{kg}^{-1}$, respectivamente, para a extração de $\mathrm{Fe}$, $\mathrm{Mn}, \mathrm{Zn}$ e Cu, por DTPA-TEA, foram excluídos para a realização das novas regressões entre os três extratores. Analisando os resultados (Quadro 3), constatou-sequetodos os $\mathrm{r}^{2}$ diminuíram, corroborando com os dados obtidos por Sims (1989) eAbreu (1997). Desta forma, para o presente estudo, a aplicação de equações de conversão entre diferentes extratores não foi recomendável.

\section{Interpretação da análise de micronutrientes}

Como as recomendações de adubação são, geralmente, orientadas pelos teores dos nutrientes determi nados na análise do sol o, resolveu-se avaliar os resultados das 103 amostras com base nos limites de interpretação em uso no Brasil, por não existir critério para o estado doRiodeJ aneiro. Em princípio, os dados foram avaliados com respeito a cinco referências de literatura mais recentes (CFCS/SC, 1994; Raij et al., 1996; Abreu et al., 1997; Alvarez V. et al., 1999; EMBRAPA, 1999) e que contemplam os três tipos de extratores utilizados no presente estudo (Quadro 4). Destas, somenteAlvarez V. et al. (1999) apresentaram mais do que três faixas de interpretação comuns, a saber: baixa, média e alta. Para fins de comparação, foram unidas as classes muito baixa e baixa em uma única classe (baixa), bem como as classes boa e alta em outra (alta).

\section{Cobre}

De maneira geral, verificou-se que, usando os limites de Raij et al. (1996), para o DTPA-TEA, encontrou-seuma maior freqüência de dados na dasse média. Já com os limites de Abreu et al. (1997), EMBRAPA (1999) e Alvarez V. et al.(1999), para o Mehlich-1, observou-se que a maioria dos dados concentra-se na classe baixa. Com respeito aos limites da CFCS/SC (1994), para o $\mathrm{HCl} 0,1 \mathrm{~mol} \mathrm{~L}^{-1}$, a mai oria dos dados está na classe alta. $\mathrm{O}$ evidente contraste desses resultados ressalta o grande problema oriundo da aplicação de classes de interpretação geradas em condições regionais diferentes das amostras analisadas.

\section{Zinco}

De maneira geral, todas as distribuições foram concordantes, apontando que a maioria dos resultados encontra-se na classe alta.

\section{Manganês}

Não houve níveis de interpretação para a extração com $\mathrm{HCl} 0,1 \mathrm{~mol} \mathrm{~L}^{-1}$ pela referência usada (CFCS, 1994). As outras também foram concordantes, indicando maior concentração de valores na dasse alta.

Ferro

Não houve níveis de interpretação para a extração com HCl 0,1 mol L-1 pela referência usada (CFCS/SC, 1994) e, no caso da extração por Mehlich-1, só uma das referências faz a citação (Alvarez V. et al., 1999). Usando os limites de Raij et al. (1996), para o DTPATEA, encontrou-semaior freqüência dedados na dasse alta.J á com os limites deAlvarezV. et al.(1999), para o M ehlich-1, observou-se mel hor distribuição eqüitativa dos dados por todas as três classes, embora cerca da metade deles esteja na classe alta. 
Quadro 3. Declividades e interceptos da regressão linear $\left(Y=b_{0}+b_{1} X\right)$ estimados pelos três extratores em 85, 42 e 21 amostras para $\mathrm{Cu}, \mathrm{Zn}$ e Mn, respectivamente

\begin{tabular}{|c|c|c|c|c|c|c|c|c|}
\hline \multirow{2}{*}{$\mathbf{Y}$} & \multirow{2}{*}{$x$} & \multicolumn{3}{|c|}{ Declividade $\left(\mathbf{b}_{1}\right)$} & \multicolumn{3}{|c|}{ Intercepto $\left(b_{0}\right)$} & \multirow{2}{*}{$\mathbf{R}^{2}$} \\
\hline & & Mínimo & Médio & Máximo & Mínimo & Médio & Máximo & \\
\hline $\mathrm{Cu}^{(2)}$ & $\mathrm{Cu}^{(1)}$ & 1,77 & 5,14 & 8,52 & $-1,82$ & $-0,70$ & 0,42 & $0,10 *$ \\
\hline $\mathrm{Cu}^{(3)}$ & $\mathrm{Cu}^{(1)}$ & 2,43 & 3,00 & 3,57 & $-0,13$ & 0,05 & 0,24 & $0,57 *$ \\
\hline $\mathrm{Cu}^{(3)}$ & $\mathrm{Cu}^{(2)}$ & 0,01 & 0,06 & 0,11 & 0,71 & 0,86 & 1,00 & $0,07 *$ \\
\hline$Z n^{(2)}$ & $\mathrm{Zn}^{(1)}$ & 0,20 & 0,76 & 1,32 & $-0,03$ & 0,61 & 1,25 & $0,16 *$ \\
\hline $\mathrm{Zn}(3)$ & $\mathrm{Zn}^{(1)}$ & 1,22 & 2,34 & 3,46 & $-2,78$ & $-1,50$ & $-0,21$ & $0,31 *$ \\
\hline $\mathrm{Zn}^{(3)}$ & $\mathrm{Zn}^{(2)}$ & 0,01 & 0,68 & 1,35 & $-0,97$ & 0,09 & 1,15 & $0,09 *$ \\
\hline$M n^{(2)}$ & $M n^{(1)}$ & $-11,50$ & $-2,46$ & 6,57 & $-8,87$ & 14,10 & 37,07 & $0,02^{\mathrm{ns}}$ \\
\hline$M n^{(3)}$ & $M n^{(1)}$ & 1,29 & 1,76 & 2,22 & $-1,50$ & $-0,32$ & 0,86 & $0,77 *$ \\
\hline$M n^{(3)}$ & $M n^{(2)}$ & $-0,06$ & $-0,01$ & 0,04 & 2,30 & 3,61 & 4,92 & $0,01^{\text {ns }}$ \\
\hline
\end{tabular}

(1) Mehlich-1. ${ }^{(2)}$ DTPA-TEA. ${ }^{(3)} \mathrm{HCl} 0,1$ mol L ${ }^{-1}$. ${ }^{*}$ Significativo a $\mathrm{P}<0,05$ e ns não-significativo.

Quadro 4. Distribuição (\%) dos resultados analíticos (expressos em $\mathrm{mg} \mathrm{kg}^{-1}$ ) das 103 amostras de solos do Rio de J aneiro, considerando algumas das classes de interpretação, baseadas nas extrações com DTPA-TEA, Mehlich-1 (M-1) e HCl, em uso no Brasil

\begin{tabular}{|c|c|c|c|c|c|c|c|c|}
\hline & DTPA $^{(1)}$ & $\%$ & $M-1^{(2)}$ & $\%$ & $\mathrm{HCl}^{(3)}$ & $\%$ & $M-\mathbf{1}^{(4)}$ & $\%$ \\
\hline \multicolumn{9}{|l|}{ Cobre } \\
\hline $\begin{array}{l}\text { Baixo } \\
\text { Médio } \\
\text { Alto }\end{array}$ & $\begin{array}{l}\leq 0,2 \\
0,3-0,8 \\
>0,8\end{array}$ & $\begin{array}{l}20,4 \\
50,5 \\
29,1\end{array}$ & $\begin{array}{l}\leq 0,4 \\
0,5-0,8 \\
>0,8\end{array}$ & $\begin{array}{l}63,1 \\
21,4 \\
15,5\end{array}$ & $\begin{aligned}<0,15 \\
\quad 0,15-0,40 \\
>0,40\end{aligned}$ & $\begin{array}{r}5,8 \\
9,7 \\
84,5\end{array}$ & $\begin{array}{l}<0,8 \\
0,8-1,2 \\
>1,2\end{array}$ & $\begin{array}{r}82,5 \\
10,7 \\
6,8\end{array}$ \\
\hline \multicolumn{9}{|l|}{ Zinco } \\
\hline $\begin{array}{l}\text { Baixo } \\
\text { Médio } \\
\text { Alto }\end{array}$ & $\begin{array}{l}\leq 0,5 \\
0,6-1,2 \\
>1,2\end{array}$ & $\begin{array}{r}2,9 \\
16,5 \\
80,6\end{array}$ & $\begin{aligned} & 1,0 \\
& 1,1-1,6 \\
> & 1,6\end{aligned}$ & $\begin{array}{l}15,5 \\
27,2 \\
57,3\end{array}$ & $\begin{aligned}<0,20 \\
\quad 0,20-0,50 \\
>0,50\end{aligned}$ & $\begin{array}{r}17,5 \\
2,9 \\
79,6\end{array}$ & $\begin{aligned}< & 1,0 \\
& 1,0-1,5 \\
> & 1,5\end{aligned}$ & $\begin{array}{l}12,7 \\
25,2 \\
62,1\end{array}$ \\
\hline \multicolumn{9}{|c|}{ Manganês } \\
\hline $\begin{array}{l}\text { Baixo } \\
\text { Médio } \\
\text { Alto }\end{array}$ & $\begin{array}{l}\leq 1,2 \\
1,3-5,0 \\
>5,0\end{array}$ & $\begin{array}{r}1,9 \\
22,3 \\
75,7\end{array}$ & $\begin{aligned} \leq & 1,9 \\
& 2,0-5,0 \\
> & 5,0\end{aligned}$ & $\begin{array}{l}10,7 \\
12,6 \\
76,7\end{array}$ & $\begin{array}{l}-- \\
-- \\
--\end{array}$ & $\begin{array}{l}-- \\
-- \\
--\end{array}$ & $\begin{array}{l}<6 \\
6-8 \\
>8\end{array}$ & $\begin{array}{l}23,3 \\
11,7 \\
65,0\end{array}$ \\
\hline \multicolumn{9}{|l|}{ Ferro } \\
\hline $\begin{array}{l}\text { Baixo } \\
\text { Médio } \\
\text { Alto }\end{array}$ & $\begin{array}{l}\leq 4 \\
5-12 \\
>12\end{array}$ & $\begin{array}{r}0,0 \\
2,9 \\
97,1\end{array}$ & $\begin{array}{l}-- \\
-- \\
--\end{array}$ & $\begin{array}{l}-- \\
-- \\
--\end{array}$ & $\begin{array}{l}-- \\
-- \\
--\end{array}$ & $\begin{array}{l}-- \\
-- \\
--\end{array}$ & $\begin{array}{l}<19 \\
19-30 \\
>30\end{array}$ & $\begin{array}{l}20,4 \\
27,2 \\
52,4\end{array}$ \\
\hline
\end{tabular}

(1) Raij et al. (1996). (2) Galrão, citado por Abreu et al. (1997) e EMBRAPA (1999). ${ }^{(3)}$ CFCS/SC (1994). ${ }^{(4)}$ Adaptado de Alvarez V. et al. (1999).

\section{CONCLUSÕES}

1. O extrator $\mathrm{HCl} 0,1 \mathrm{~mol} \mathrm{~L}^{-1}$ foi oqueapresentou maior capacidade de extração de $\mathrm{Cu}, \mathrm{Fe}, \mathrm{Zn}$ e Mn, quando comparado com o DTPA-TEA e o M ehlich-1.

2. Pelos resultados encontrados, verificou-se, para os diferentes extratores, o predomínio da faixa de disponibilidade alta para a maioria dos micronutrientes estudados, sendo exceção o cobre que apresentou predomínio da faixa de disponi bilidade baixa.
3. Equações de conversão entre os teores dos diferentes extratores testados não apresentaram ajustesatisfatório, indicando queval ores obti dos com um extrator não podem ser convertidos em valores estimados para outro extrator.

4. Faixas deinterpretação, principalmenteno caso do $\mathrm{Cu}$ e do $\mathrm{Fe}$, desenvolvidas em outras regiões, de características edáficas bem diferentes das encontradas noestado do Rio deJ aneiro, podem levar a sérios erros de diagnose da fertilidade quanto a esses el ementos. 


\section{AGRADECIMENTOS}

AoCNPq-PIBIC, pela bolsa del niciaçãoCientífica concedida, eao convênio entrea UFRRJ ea EmbrapaSolos, que permitiu ouso das 103 amostras estudadas.

\section{LITERATURA CITADA}

ABREU, C.A. Análise de solo para micronutrientes - Tema de reuniões de laboratórios. B. Inf. SBCS, 20:128-130, 1995.

ABREU, C.A.; LOPES, A.S. \& RAIJ, B. van. Análise de micronutrientes em solos brasileiros: situação atual e perspectiva. In: CONGRESSO BRASI LEIRO DE CIÊNCIA DO SOLO, 26., Rio de J aneiro, 1997. Anais. Rio de J aneiro, Empresa Brasileira de Pesquisa Agropecuária, 1997. 20p. (CD-ROM)

ALVA, A.K. Comparision of Mehlich-3, Mehlich-1, ammonium bicarbonate-DTPA, 1.0M acetate and 0.2M ammonium chloride for extraction of calcium, magnesium, phosphorus and potassium for a wide range of soils. Comm. Soil Sci. Plant Anal., 24:603-6012, 1993.

ALVAREZV., V.H.; NOVAIS, R.F.; BARROS, N.F.; CANTARUTTI, R.B.; LOPES, A.S. I nterpretação dos resultados das análises de solos. In: COMISSÃO DE FERTILIDADE DO SOLO DO ESTADO DE MINAS GERAIS. Recomendações para o uso de corretivos e fertilizantes em Minas Gerais. Viçosa, 1999. p.25-32.

ASSUMPÇÃO, J.C. Comparação dos extratores EDTA, DTPA e acetato de amônio ( $\mathrm{NH}_{4} \mathrm{Oac}$ ) com o extrator Mehlich na determinação de micronutrientes em solos tropicais. Niterói, Universidade Federal Fluminense, 1995. 118p. (Tese de Mestrado)

BATAGLIA, O.C. \& RAIJ, B. van. Eficiência de extratores de micronutrientes na análise de sol o. R. Bras. Ci. Solo, 13:205212, 1989.

CAMARGO, O.A. Micronutrientes no solo. In: BORKERT, C.M. \& LANTMANN, A.F., eds. Enxofre e micronutrientes na agricultura brasileira. Londrina, Empresa Brasileira de Pesquisa Agropecuária, 1988. p.103-120.

CANTARELLA, H.; QUAGGIO, J.A.; MATTOS J UNIOR, D. A análise de solo no Brasil: 1982-1989. B. Inf. SBCS, 13:96112, 1994.

CANTARELLA, H.; RAIJ , B. van. \& QUAGGIO, J.A. Soil and plant analyses for lime and fertilizer recommendations in Brazil. Comm. Soil Sci. Plant Anal., 29:1691-1706, 1998.

COMISSÃO DE FERTILIDADE DO SOLO-CFRS/SC. Recomendações de adubação e calagem para os estados do Rio Grande do Sul e Santa Catarina. 2.ed. Passo Fundo: Núcleo Regional Sul, 1994. 224p.

ECKERT, D.J. \& WATSON, M.E. Integrating the Mehlich-3 extractant into existing soil test interpretation schemes. In: HOOD, T.M. \& J ONES J r., J .B., eds. Soil and plant analysis in sustainable agriculture and environment. New York, Marcel Dekker, 1997. p.239-251.

EMPRESA BRASILEIRA DE PESQUISA AGROPECUÁRIA EMBRAPA. Centro Nacional de Pesquisa de Solos. Manual de métodos de análise de solo. Rio de J aneiro, 1997. 212p.
EMPRESA BRASILEIRA DE PESQUISA AGROPECUÁRIA EMBRAPA. Centro Nacional de Pesquisa de Solos. Sistema brasileiro de classificação de solos. Brasília, Produção de Informação; Rio de J aneiro, Embrapa Solos, 1999. 412p.

EMPRESA BRASILEIRA DE PESQUISA AGROPECUÁRIA EMBRAPA. Recomendações técnicas para a cultura da soja no Paraná 1999/2000. Londrina, Embrapa Soja, 1999. 236p.

FERREIRA, M.E. \& CRUZ, M.C.P.Simpósio sobremicronutrientes na agricultura, 1., J aboticabal, 1988. Anais. Piracicaba, POTAFOS/CNPq, 1991. 734p.

FREIRE, L.R.; BLOISE, R.M.; MOREIRA, G.N.C. \& EIRA, P.A. Análise química do solo. In: De-POLLI, H., coord. Manual de adubação para o Rio de J aneiro. Itaguaí, Universidade Rural, 1988. p.24-37.

GARCÍA, A.; IORIO, A.F.; BARROS, M.; BARGIELA, M. \& RENDINA, A. Comparison of soil tests to determine micronutrients status in Argentina soils. Comm. Soil Sci. Plant Anal., 28:1777-1792, 1997.

J ONES, U.S. \& PIHA, M. Evaluation of four test extractants for Zimbabwe soils. Commun. Soil Sci. Plant Anal., 20:18571871, 1989.

LINDSAY, W.L. \& NORVELL,W.A. Development of a DTPA soil test for zinc, iron manganese and copper. Soil Sci. Soc. Am. J., 42:421-428, 1978.

MAMO, T.; RICHTER, C. \& HEILIGTAG, B. Comparison of extractants for the determination of available phosphorus, potassium, calcium, magnesium and sodium in some ethiopian and german soils. Comm. Soil Sci. Plant Anal., 27:2197-2212, 1996

MILLER,J.C. \& MILLER, J.N. Statistics for analytical chemistry. 3.ed. Chichester, Ellis Horwood, 1993. p.120-124.

NELSON, J.L., BOAWN, L.C. \& VIETS J r., F.G. A method for assessing zinc status of soils using acid-extractable zinc and “titratablealkalinity" values. Soil Sci., 88:275-283, 1959.

NELSON, W.L.; MEHLICH,A.\& WINTERS, E. The development, evaluation, and use of soil tests for phosphorus availability. In: PIERRE, W.H. \& NORMAN, A.G., eds. Soil and fertilizer phosphorus. New York, Academic Press, 1953. v.4, p.153188.

NIE, N.H.; HULL, C.H.; J ENKINS, J .G.; STEINBRENNER, K. \& BENT, D.H. Statistical package for the social sciences. 2.ed. Chicago, McGraw-Hill, 1979. 675p.

RAIJ , B. van; CANTARELLA, H.; QUAGGIO, J .A. \& FURLANI, A.M.C., eds. Recomendações de adubação e calagem para o estado de São Paulo. 2.ed. Campinas, Instituto Agronômico \& Fundação IAC, 1996. 285p.

RIBEIRO, A.C. \& SARABIA, T.W.A. Avaliação de extratores para zinco e boro disponíveis em latossol os do triângulo mineiro. R. Bras. Ci. Solo, 8:85-89, 1984.

SIMS, J.T. Comparison of Mehlich 1 and Mehlich 3 extractants for $\mathrm{P}, \mathrm{K}, \mathrm{Ca}, \mathrm{Mg}, \mathrm{Mn}, \mathrm{Cu}$ and $\mathrm{Zn}$ in Atlantic Coastal Plain Soils. Comm. Soil Sci. Plant Anal., 20:1707-1726, 1989.

WOLF,A.M. \& BAKER, D.E. Comparisions of soil test phosphorus by Olsen, Bray P1, Mehlich-1 and Mehlich-3 methods. Comm. Soil Sci. Plant Anal., 16:467-484, 1985. 